\title{
A Common Fixed Point Theorem for a Sequence of Multivalued Mappings
}

By

\author{
Kenjiro YANAGI*
}

\section{$\S 1$ Introduction}

Recently, fixed point theorems for multivalued contraction mappings in metric spaces were obtained by many authors ([5], [2], [7], [1], [4], [6], etc). On the other hand, Dube [3] proved a theorem on common fixed points of two multivalued mappings.

In this paper, we shall give a common fixed point theorem for a sequence of multivalued mappings satisfying some conditions in complete metric spaces. In our theorem, we shall obtain an extension of the result of Itoh [4] to the case of a sequence of multivalued mappings.

\section{$\S$ 2. Preliminaries}

Let $(X, d)$ be a metric space. For any $x \in X$ and $A \subset X$, we define $D(x, A)=\inf \{d(x, y): y \in A\}$. Let $C B(X)$ denote the family of all nonempty closed bounded subsets of $X$. For $A, B \in C B(X)$, let $H(A, B)$ denote the distance between $A$ and $B$ in the Hausdorff metric induced by $d$ on $C B(X)$. The following lemmas are direct consequences of definition of Hausdorff metric.

Lemma 1. If $A, B \in C B(X)$ and $x \in A$, then $D(x, B) \leqq H(A, B)$.

Lemma 2. For any $x \in X, A, B \in C B(X)$,

$$
|D(x, A)-D(x, B)| \leqq H(A, B) .
$$

Lemma 3. Let $A, B \in C B(X)$ and $k \in(1, \infty)$ be given. Then for

Communicated by S. Matsuura, June 24, 1976. Revised July 1, 1977.

* Department of Mathematics, Yamaguchi University. 
$a \in A$, there exists $b \in B$ such that $d(a, b) \leqq k H(A, B)$.

$(X, d)$ is said to be metrically convex if for any $x, v \in X$ with $x$ $\neq y$, there exists an element $z \in X, x \neq z \neq y$, such that

$$
d(x, \approx)+d(z, y)=d(x, y) .
$$

In Assad and Kirk [1], the following is noted.

Lemma 4. If $K$ is a nonempty closed subset of the complete and metrically convex metric space $(X, d)$, then for any $x \in K, y \notin K$, there exists $\approx \in \partial K$ (the boundary of $K$ ) such that

$$
d(x, z)+d(z, y)=d(x, y) .
$$

\section{$\S$ 3. A Common Fixed Point Theorem}

The following is a common fixed point theorem for a sequence of multivalued mappings $T_{n}: K \rightarrow C B(X)$ when $K$ is a nonempty closed subset of a complete metrically convex metric space $X$.

Theorem. Let $(X, d)$ be a complete and metrically convex metric space and $K$ be a nonempty closed subset of $X$. Let $T_{n}(n=1$, $2, \cdots)$ be a sequence of multivalued mappings of $K$ into $C B(X)$. Suppose that there are nonnegative real numbers $\alpha, \beta, \gamma$ with $\alpha+$ $(\alpha+3)(\beta+\gamma)<1$ such that

$$
\begin{aligned}
H\left(T_{i}(x), T_{j}(y)\right) \leqq \alpha d(x, y) & +\beta\left\{D\left(x, T_{i}(x)\right)+D\left(y, T_{j}(y)\right)\right\} \\
& +\gamma\left\{D\left(x, T_{j}(y)\right)+D\left(y, T_{i}(x)\right)\right\}
\end{aligned}
$$

for all $x, y \in K$ and for all $i, j=1,2, \cdots$. If $T_{n}(x) \subset K$ for each $x$ $\in \partial K$ and each $n=1,2, \cdots$, then the sequence $T_{n}(n=1,2, \cdots)$ has a common fixed point in $K$.

Proof. Since $Y$ is metrically convex, there exists $x_{0} \in K$ such that $T_{1}\left(x_{0}\right) \subset K$. If $\alpha=\beta=\gamma=0$, any $\approx \in T_{1}\left(x_{0}\right)$ is a common fixed point. In fact, for $n=2,3, \cdots, H\left(T_{1}\left(x_{0}\right), T_{n}(z)\right)=0$ and then $z \in T_{n}(z), n=2,3, \cdots$. Since $H\left(T_{1}(z), T_{2}(z)\right)=0$, we have $z \in T_{1}(z)$. So we assume that 
$c \alpha+\beta+\gamma>0$. Since $c \alpha+(\alpha+3)(\beta+\gamma)<1$, there exists $k>1$ such that $(1+k \beta+k \gamma)(k \alpha+k \beta+k \gamma) /(1-k \beta-k \gamma)^{2}<1$. We choose sequences $\left\{x_{n}\right\}$ in $K$ and $\left\{y_{n}\right\}$ in the following way. Let $x_{1}=y_{1} \in T_{1}\left(x_{0}\right)$. By Lemma 3 , there exists $y_{2} \in T_{2}\left(x_{1}\right)$ such that $d\left(y_{1}, y_{2}\right) \leqq k H\left(T_{1}\left(x_{0}\right), T_{2}\left(x_{1}\right)\right)$. If $y_{2} \in K$, let $x_{2}=y_{2}$. If $y_{2} \notin K$, choose an element $x_{2} \in \partial K$ such that $d\left(x_{1}, x_{2}\right)+d\left(x_{2}, y_{2}\right)=d\left(x_{1}, y_{2}\right)$ by Lemma 4 . By introduction we can obtain sequences $\left\{x_{n}\right\},\left\{y_{n}\right\}$ such that for $n=1,2, \cdots$,

(a) $y_{n+1} \in T_{n+1}\left(x_{n}\right)$,

(b) $d\left(y_{n}, y_{n+1}\right) \leqq k H\left(T_{n}\left(x_{n-1}\right), T_{n-1}\left(x_{n}\right)\right)$,

where

(c) if $y_{n+1} \in K$, then $x_{n+1}=y_{n+1}$, and

(d) if $y_{n+1} \notin K$, then $x_{n+1} \in \partial K$ and

$$
d\left(x_{n}, x_{n+1}\right)+d\left(x_{n-1}, y_{n}\right)=d\left(x_{n}, y_{n+1}^{\prime}\right) .
$$

We shall estimate the distance $d\left(x_{n}, x_{n+1}\right)$ for $n \geqq 2$. There arise three cases.

(i) The case that $x_{n}=y_{n}$ and $x_{n+1}=y_{n+1}$. We have

$$
\begin{aligned}
& d\left(x_{n}, x_{n+1}\right)=d\left(y_{n}, y_{n+1}\right) \\
& \leqq k H\left(T_{n}\left(x_{n-1}\right), T_{n+1}\left(x_{n}\right)\right) \\
& \leqq k \alpha d\left(x_{n-1}, x_{n}\right)+k \beta\left\{D\left(x_{n-1}, T_{n}\left(x_{n-1}\right)\right)+D\left(x_{n}, T_{n+1}\left(x_{n}\right)\right)\right\} \\
& \quad+k \gamma\left\{D\left(x_{n-1}, T_{n+1}\left(x_{n}\right)\right)+D\left(x_{n}, T_{n}\left(x_{n-1}\right)\right)\right\} \\
& \leqq k \alpha d\left(x_{n-1}, x_{n}\right)+k \beta\left\{d\left(x_{n-1}, x_{n}\right)+d\left(x_{n}, x_{n+1}\right)\right\} \\
& +k r\left\{d\left(x_{n-1}, x_{n}\right)+d\left(x_{n}, x_{n+1}\right)\right\} .
\end{aligned}
$$

Hence

$$
(1-k \beta-k \gamma) d\left(x_{n}, x_{n+1}\right) \leqq(k \alpha+k \beta+k \gamma) d\left(x_{n-1}, x_{n}\right)
$$

and

$$
d\left(x_{n}, x_{n+1}\right) \leqq \frac{k \alpha+k \beta+k \gamma}{1-k \beta-k \gamma} d\left(x_{n-1}, x_{n}\right) .
$$

(ii) The case that $x_{n}=y_{n}$ and $x_{n+1} \neq y_{n\lrcorner 1}$. By (d) we obtain that

$$
d\left(x_{n}, x_{n-1}\right) \leqq d\left(x_{n}, y_{n+1}\right)=d\left(y_{n}, y_{n+1}^{\prime}\right) .
$$

As in the case (i), we have 


$$
d\left(y_{n}, y_{n+1}\right) \leqq \frac{k \alpha+k \beta+k \gamma}{1-k \beta-k \gamma} d\left(x_{n-1}, x_{n}\right)
$$

thus

$$
d\left(x_{n}, x_{n+1}\right) \leqq \frac{k \alpha+k \beta+k \gamma}{1-k \beta-k \gamma} d\left(x_{n-1}, x_{n}\right)
$$

(iii) The case that $x_{n} \neq y_{n}$ and $x_{n+1}=y_{n+1}$. In this case $x_{n-1}=y_{n-1}$ holds. We have

$$
\begin{aligned}
d\left(x_{n}, x_{n+1}\right) & \leqq d\left(x_{n}, y_{n}\right)+d\left(y_{n}, x_{n+1}\right) \\
& =d\left(x_{n}, y_{n}\right)+d\left(y_{n}, y_{n+1}\right) .
\end{aligned}
$$

From (b) it follows that

$$
\begin{aligned}
& d\left(y_{n}, y_{n+1}\right) \leqq k H\left(T_{n}\left(x_{n-1}\right), T_{n+1}\left(x_{n}\right)\right) \\
& \leqq k \alpha d\left(x_{n-1}, x_{n}\right)+k \beta\left\{D\left(x_{n-1}, T_{n}\left(x_{n-1}\right)\right)+D\left(x_{n}, T_{n+1}\left(x_{n}\right)\right)\right\} \\
& +k \gamma\left\{D\left(x_{n-1}, T_{n+1}\left(x_{n}\right)\right)+D\left(x_{n}, T_{n}\left(x_{n-1}\right)\right)\right\} \\
& \leqq k \alpha d\left(x_{n-1}, x_{n}\right)+k \beta\left\{d\left(x_{n-1}, y_{n}\right)+d\left(x_{n}, x_{n+1}\right)\right\} \\
& +k r\left\{d\left(x_{n-1}, x_{n}\right)+d\left(x_{n}, x_{n+1}\right)+d\left(x_{n}, y_{n}\right)\right\} \text {. }
\end{aligned}
$$

Hence we obtain

$$
\begin{gathered}
d\left(x_{n}, x_{n+1}\right) \leqq(1+k \gamma) d\left(x_{n}, y_{n}\right)+(k \alpha+k \gamma) d\left(x_{n-1}, x_{n}\right) \\
+k \beta d\left(x_{n-1}, y_{n}\right)+(k \beta+k \gamma) d\left(x_{n}, x_{n+1}\right) \\
\leqq(1+k \gamma) d\left(x_{n-1}, y_{n}\right)+k \beta d\left(x_{n-1}, y_{n}\right)+(k \beta+k \gamma) d\left(x_{n}, x_{n+1}\right),
\end{gathered}
$$

which implies

$$
d\left(x_{n}, x_{n+1}\right) \leqq \frac{1+k \beta+k r}{1-k \beta-k r} d\left(x_{n-1}, y_{n}\right) .
$$

Since $x_{n-1}=y_{n-1}$ and $x_{n} \neq y_{n}$, it follows from (ii) that

$$
d\left(x_{n-1}, y_{n}\right) \leqq \frac{k \alpha+k \beta+k \gamma}{1-k \beta-k \gamma} d\left(x_{n-2}, x_{n-1}\right) \text {. }
$$

Thus it follows that for every $n \geqq 2$,

$$
d\left(x_{n}, x_{n+1}\right) \leqq \frac{(1+k \beta+k \gamma)(k \alpha+k \beta+k \gamma)}{(1-k \beta-k \gamma)^{2}} d\left(x_{n-2}, x_{n-1}\right)
$$

The case that $x_{n} \neq y_{n}$ and $x_{n-1} \neq y_{n+1}$ does not occur. Since 


$$
\frac{k \alpha+k \beta+k \gamma}{1-k \beta-k \gamma} \leqq \frac{(1+k \beta+k \gamma)(k \alpha+k \beta+k \gamma)}{(1-k \beta-k \gamma)^{2}},
$$

we proved the following; for $n \geqq 2$,

$$
d\left(x_{n}, x_{n+1}\right) \leqq\left\{\begin{array}{l}
p d\left(x_{n-1}, x_{n}\right), \text { or } \\
p d\left(x_{n-2}, x_{n-1}\right),
\end{array}\right.
$$

where $p=(1+k \beta+k \gamma)(k \alpha+k \beta+k \gamma) /(1-k \beta-k \gamma)^{2}$. Put

$$
\delta=p^{-1 / 2} \max \left\{d\left(x_{0}, x_{1}\right), d\left(x_{1}, x_{2}\right)\right\},
$$

then by induction we can show that

$$
d\left(x_{n}, x_{n+1}\right) \leqq p^{n / 2} \delta \quad(n=1,2, \cdots) .
$$

It follows that for any $m>n \geqq 1$,

$$
d\left(x_{n}, x_{m}\right) \leqq 0 \sum_{i=n}^{m-1} p^{i / 2} .
$$

This implies that $\left\{x_{n}\right\}$ is a Cauchy sequence. Since $X$ is complete and $K$ is closed, $\left\{x_{n}\right\}$ converges to some point $z \in K$. From the definition of $\left\{x_{n}\right\}$, there exists a subsequence $\left\{x_{n_{i}}\right\}$ of $\left\{x_{n}\right\}$ such that $x_{n_{i}}=y_{n_{i}}$ $(i=1,2, \cdots)$. Then for $n=1,2, \cdots$, we have

$$
\begin{aligned}
& D\left(x_{n_{i}}, T_{n}(z)\right) \leqq H\left(T_{n_{i}}\left(x_{n_{i}-1}\right), T_{n}(\approx)\right) \\
& \leqq \alpha d\left(x_{n_{i}-1}, z\right)+ \beta\left\{D\left(x_{n_{i}-1}, T_{n_{i}}\left(x_{n_{i}-1}\right)\right)+D\left(z, T_{n}(z)\right)\right\} \\
&+\gamma\left\{D\left(x_{n_{i}-1}, T_{n}(z)\right)+D\left(z, T_{n_{i}}\left(x_{n_{i}-1}\right)\right)\right\} \\
& \leqq \alpha\left\{d\left(x_{n_{i}-1}, x_{n_{i}}\right)+d\left(x_{n_{i}}, z\right)\right\} \\
&+\beta\left\{d\left(x_{n_{i}-1}, x_{n_{i}}\right)+d\left(\approx, x_{n_{i}}\right)+D\left(x_{n_{i}}, T_{n}(\approx)\right)\right\} \\
&+\left.\gamma d\left(x_{n_{i}-1}, x_{n_{i}}\right)+D\left(x_{n_{i}}, T_{n}(z)\right)+d\left(z, x_{n_{i}}\right)\right\} .
\end{aligned}
$$

Thus

$$
D\left(x_{n_{i}}, T_{n}(z)\right) \leqq \frac{\alpha+\beta+\gamma}{1-\beta-\gamma}\left\{d\left(. x_{n_{2}-1}, x_{n_{i}}\right)+d\left(z, x_{n_{i}}\right)\right\} .
$$

Therefore, $D\left(x_{n_{i}}, T_{n}(z)\right) \rightarrow 0$ as $i \rightarrow \infty$. By the inequality $D\left(z, T_{n}(\approx)\right)$ $\leqq d\left(x_{n_{i}}, z\right)+D\left(x_{n_{i}}, T_{n}(z)\right)$ and the above result, it follows that $D\left(z, T_{n}(z)\right)=0$. Since $T_{n}(z)$ is closed, this implies that $z \in T_{n}(z)$, $n=1,2, \cdots$. 
Remark. If $\alpha, \beta, \gamma \geqq 0$, then $\alpha+(\alpha+3)(\beta+\gamma)<1$ if and only if $(1+\beta+\gamma)(\alpha+\beta+\gamma) /(1-\beta-\gamma)^{2}<1$. Hence putting $T_{n}=T$ for $n=$ $1,2, \cdots$ in our theorem, we obtain the result of Itoh [4].

Since every Banach space is metrically convex, we have the following corollary for singlevalued mappings.

Corollary. Let $E$ be a Banach space and $K$ be a nonempty closed subset of $E$. Let $f_{n} \quad(n=1,2, \cdots)$ a sequence of singlevalued mappings of $K$ into $E$. Suppose that there are nonnegative real numbers $\alpha, \beta$, $\gamma$ with $\alpha+(\alpha+3)(\beta+\gamma)<1$ such that

$$
\begin{aligned}
\left\|f_{i}(x)-f_{j}(y)\right\| & \leqq \alpha\|x-y\|+\beta\left\{\left\|x-f_{i}(x)\right\|+\left\|y-f_{j}(y)\right\|\right\} \\
& +\gamma\left\{\left\|x-f_{j}(y)\right\|+\left\|y-f_{i}(x)\right\|\right\}
\end{aligned}
$$

for all $x, y \in K$ and for all $i, j=1,2, \cdots$. If $f_{n}(\partial K) \subset K$ for each $n$ $=1,2, \cdots$, then there exists an unique common fixed point $\approx \in K$.

We conclude this paper by stating an open problem whether our theorem holds when nonnegative real numbers $\alpha, \beta, \gamma$ satisfy $\alpha+2 \beta+2 \gamma$ $<1$ instead of $\alpha+(\alpha+3)(\beta+\gamma)<1$.

Acknowledgement. The author wishes to thank the referee for his critical and helpful comments.

\section{References}

[1] Assad, N. A. and Kirk, W. A., Fixed point theorems for set-valued mappings of contractive type, Pacific J. Math., 43 (1972), 553-562.

[2] Dube, L. S. and Singh, S. P., On multivalued contraction mappings, Bull. Math. Soc. Sci. Math. R. S. Roumanie, 14 (1970), 307-310.

[3] Dube, L. S., A theorem on common fixed points of multivalued mappings, Ann. Soc. Sci. Bruxelles, 89 (1975), 463-468.

[4] Itoh, S., Multivalued generalized contractions and fixed point theorems, Research Reports on Information Sciences, Tokyo Institute of Technology, A-21 (1975) .

[5] Nadler, S. B. Jr., Multivalued contraction mappings, Pacific J. Math., 30 (1969), 457-488.

[6] Ray, B. K., Some fixed point theorems, Fund. Math., XCII (1976), 76-90.

[7] Reich, S., Kannan's fixed point theorem, Bollettino U. M. I., 4 (1971), 1-11. 